\title{
La articulación, requisito básico de un producto de investigación educativa
}

\section{Linkage: Basic Requirement in any Product of Research in Education}

\author{
Yolanda González de la Torre ${ }^{l}$ \\ Departamento de Estudios en Educación \\ Universidad de Guadalajara \\ Guadalajara, México \\ yoly1565@yahoo.com.mx
}

\author{
José Jiménez Mora ${ }^{2}$ \\ Centro de Bachillerato Tecnológico, industrial y de servicios No. 10 \\ Secretaría de Educación Pública \\ México \\ pepe_dw@hotmail.com
}

Recibido 27 de agosto de 2011 • Aceptado 23 de febrero 2012

\begin{abstract}
Resumen. Dentro de la producción científica en el campo de la educación se hace constante referencia a la articulación como una condición esencial en el desarrollo de una investigación. No obstante, el significado de esta condición y la forma de conseguirla es un asunto que no siempre queda claro, sobre todo para quienes se encuentran en una etapa de formación en la investigación educativa. Mientras, quienes fungen como lectores y lectoras que realizan crítica de la producción académica señalan la articulación como aspecto relevante y la solicitan como rasgo fundamental en cualquier trabajo de investigación. Para aportar a la discusión sobre este aspecto, la reflexión de este trabajo propone que la articulación comprende, por una parte, una reflexión epistemológica sobre los componentes de la investigación en cada una de sus etapas, como la construcción del objeto de estudio, la definición de la metodología, el análisis de los datos y su interpretación, entre las más importantes. Por otra parte, la articulación se cristaliza en un producto escrito que refleja los resultados de una construcción y comunicación ordenada de pensamiento, acorde con las reglas establecidas en un campo de conocimiento.
\end{abstract}

Palabrasclaves. Articulación, investigación educativa, epistemología,redacción académica, metodología.

\footnotetext{
Doctora en Educación por la Universidad de Guadalajara. Ha sido jefa del Departamento de Fomento a la Lectura y, posteriormente, coordinadora operativa en la Red Estatal de Bibliotecas del Estado de Jalisco. Actualmente se desempeña como profesora-investigadora en el Departamento de Estudios en Educación de la Universidad de Guadalajara. Imparte cursos en pregrado y posgrado de metodología de la investigación y seminarios sobre lectura. Temas de investigación que trabaja: Procesos lectores, evaluación de la lectura, tecnocultura y producción de conocimiento. Sus trabajos han sido publicados en: Perfiles educativos, revista de la Universidad Nacional Autónoma de México; Revista electrónica de investigación educativa (Redine), de la Universidad Lisandro Alvarado, Venezuela; y en las editoriales CUCSH/ U DE G, y Editorial Universitaria.

2 Maestro en Investigación en Ciencias de la Educación por la Universidad de Guadalajara. Ha sido Jefe de la Unidad de Investigación del Sistema de Educación Media Superior en esta universidad y formador de docentes en el posgrado por parte de la Secretaría de Educación de Jalisco. Actualmente se desempeña como investigador asistente en el Departamento de Estudios en Educación de la Universidad de Guadalajara y como profesor en el Centro de Bachillerato Tecnológico, industrial y de servicios No. 10. Ha colaborado en la revista Educación y desarrollo de la Universidad de Guadalajara, en la revista Perfiles educativos, de la Universidad Nacional Autónoma de México, y en la revista Mexicana de investigación educativa. Así mismo, ha participado en publicaciones de la Universidad de Guadalajara, relacionadas con los temas de la investigación educativa. Es coautor de la obra Maltrato entre alumnos. El lado oscuro de la escuela, publicada por la editorial Novedades Educativas en este año.
} 


\begin{abstract}
The scientific production, in the field of education, constantly refers to linkage as a special condition to develop research. However, its meaning and the way to achieve that condition are not always clear, particularly for those who are in a training stage of research in education. Meanwhile, the academic production reviewers consider linkage as a very important aspect and essential characteristic of any research project. This paper suggests that linkage involves an epistemological discussion on the research factors in each one of the research stages, such as the construction of the object of study, the definition of methodology, and the data analysis and interpretation, among the most important stages. Linkage takes shape in a written product showing the results from an orderly construction and communication of thought, according to the rules established in a particular field of knowledge.
\end{abstract}

Keywords. Linkage, educational research, epistemology, academic writing, methodology.

\title{
Introducción
}

En el mundo académico existen diversos escenarios en los cuales se realizan valoraciones sobre las características que adopta la producción lograda por quienes se dedican a la investigación educativa o por quienes se encuentran en un proceso de formación para esta tarea, como los estudiantes y las estudiantes de posgrado, para quienes la formación se concreta no solo en el abordaje de los contenidos de un programa particular, sino en las experiencias vividas en la interacción con individuos considerados expertos en este campo. Entre estas experiencias, los dictámenes emitidos sobre artículos o libros, y las críticas realizadas a un proyecto de investigación, son ejemplos de valoraciones con las cuales, de alguna manera, se dan a conocer una serie de requerimientos de un trabajo de investigación, especialmente en lo que se refiere a los procesos intelectuales por los que atraviesa y a sus características como producto escrito.

Recordar esta situación remite a un conjunto de significados presentes en el discurso sobre la investigación, cuya comprensión resulta difícil demostrar al momento de concretar un producto escrito, dada la complejidad de la escritura académica. Cuestiones como la anterior han dado lugar a propuestas didácticas sobre las diversas formas de lectura y escritura en educación superior, como la de Narvaja di Arnoux, Di Stéfano y Pereira (2002), donde explican cómo y para qué se deben realizar determinadas tareas en la composición de un texto académico, como la incorporación de las aportaciones de los autores que se leen respecto a un tema. Desde la perspectiva del trabajo citado -en el ejemplo de tarea al cual se alude- las ideas de otros autores que son referidas en un texto académico deben servir de soporte argumentativo, pero es justamente la forma de incluirlas dentro del escrito la que permite distinguir si cumplen o no esta función.

En el terreno de la formación en el posgrado, una de las condiciones en las que ponen más atención quienes tienen a su cargo la revisión de un trabajo de investigación es la articulación. Esta condición hace referencia, primeramente, a la conjunción de los procesos y los contenidos que intervienen en la producción de conocimiento científico y, en segundo lugar, implica un tipo de pensamiento y escritura que permita generar un documento en el que se aprecie coherencia entre cada una de las etapas y procedimientos por los cuales atraviesa la investigación.

A nivel epistemológico, en un trabajo articulado se puede percibir una lógica de construcción donde existe correspondencia entre los conceptos y planteamientos que dan lugar a las preguntas de investigación y la metodología para encontrar información a fin de darles respuesta. Como señalan 
Bourdieu, Chamboredon y Passeron (1975), la cuestión epistemológica refiere a la confrontación permanente de la persona que realiza investigación entre sus supuestos y los procedimientos que elige para recolectar datos y analizarlos. Investigar implica, entonces, concebir una estructura unitaria en la cual hay un conjunto de componentes que se van construyendo, guardando armonía de funciones unos con otros y donde, en palabras de Zanotto (2007), la lógica de las ideas tiene una función esencial para justificar la posición del autor frente al conocimiento. Los contenidos se deben encontrar organizados lógicamente de manera que se puede presentar una idea central sustentada en datos y teorías.

En lo que se refiere a la redacción, un trabajo articulado cuenta con características que lo hacen comprensible, tales como la precisión en el tratamiento de los conceptos y el uso correcto de enlaces entre afirmaciones, párrafos, apartados y capítulos. Sobre este punto, la presentación del texto debe mostrar el cuidado de aspectos de redacción, como los referidos a reglas ortográficas y gramaticales, porque eso refleja orden en la construcción y comunicación del pensamiento.

En relación con lo anterior, conviene señalar que el documento en el cual se reporta una investigación, donde se incorporan conceptos, reflexiones y explicaciones del fenómeno estudiado, tiene que atender un propósito comunicativo definido y, de alguna forma, manifestar que hay una apropiación de la cultura de la producción de conocimiento del campo en cuestión. En este sentido, el desarrollo del proceso de escritura es un asunto de práctica social y cultural en el que la forma de expresión, organización y argumentación se tienen que ajustar a los usos establecidos y aceptados por una comunidad de producción de conocimiento (Kalman, 2000). La investigación, entonces, implica tener el dominio de un discurso que manifieste los conocimientos pertinentes de acuerdo con un campo de conocimiento, de tal manera que en la comunicación escrita se exprese el contenido respetando las reglas que privan en tal campo (Cassany y Morales, 2008).

Con la intención de concretar una reflexión que sirva para cuidar que un trabajo de investigación se encuentre articulado, los apartados que siguen exponen distintos momentos en los cuales esta condición se pone a prueba.

\section{La primera aduana: la construcción del objeto de estudio}

La expresión a la cual hace referencia este subtítulo, y los dos siguientes, en torno a las "aduanas" por las cuales tiene que pasar un producto de investigación que se socializa por escrito, es empleada de manera metafórica, desde el supuesto de que cada momento de una investigación implica, para quien la realiza, cumplir con determinados requisitos. Dicho de otra manera, durante la realización de una investigación y su puesta por escrito, existen condiciones de rigor académico que representan filtros por los cuales atraviesa el documento.

Una expresión común en el campo de la investigación educativa para referirse a la delimitación del objeto de interés de quien investiga es la construcción del objeto. Esta es una de las primeras tareas en las cuales se refleja que apropiarse de un discurso no es igual que apropiarse de su significado. Una persona puede hablar y hablar de "la construcción del objeto" sin saber cómo hacerla. Para apreciar si un objeto de estudio está construido en forma aceptable, dos de los recursos más importantes son la comparación con trabajos de investigación publicados y la revisión de los pares y los expertos. 
Con referencia a este momento de la investigación, Cervantes (1997) advierte la importancia de especificar la naturaleza de la articulación, que tiene que ver con dos aspectos:

- El ordenamiento y enlace de los elementos teóricos y las preguntas que se insertan en la discusión, el cual permite emerger un objeto de estudio.

- El desarrollo de planteamientos sobre la pertinencia del objeto y los límites de su construcción.

Cuando se lleva a cabo este proceso, aparecen cuestiones que se deben tener presentes en torno a la naturaleza del objeto de estudio, tales como los conceptos que lo definen y la(s) pregunta(s) que se genera(n) en torno al mismo; pero, sobre todo, se tienen que hacer explícitas las relaciones entre ellos, lo cual obliga a mirar de forma vinculada los conceptos que en principio se determinan de manera aislada respecto a un objeto de estudio.

Por lo que respecta a la construcción discursiva del objeto, con la llegada de los procesadores de texto y otras tecnologías de la información y comunicación, una de las tareas principales de una investigación, representada por la revisión y apropiación de referentes teóricos, ha tenido que cambiar, de las antiguas fichas de contenido, a otras posibilidades de elaboración de bases de datos más amplias y complejas. Sin embargo, tanto a través de recursos tradicionales como de los representados por estos nuevos medios tecnológicos, los procesos de tipo intelectual vinculados a la escritura de un producto como la construcción de un objeto de estudio son los mismos. Entre otros, una lectura comprensiva y cuidadosa de los textos y la localización de información útil para orientar la reflexión son dos ejemplos de lo que cualquier investigador o investigadora tiene que realizar cuando trabaja con información de diversas fuentes.

No obstante, uno de los riesgos de las actuales formas de tratamiento de la información es precisamente el de "cortar y pegar" segmentos de discurso producido por otros autores, sin que medie entre esta aglomeración de texto una serie de afirmaciones que vayan conduciendo lo que se escribe de tal manera que resulte un producto legible.

La articulación, en contraste, implica la inclusión ordenada de conceptos y afirmaciones que permita hilar de manera lógica una temática de discusión dada. Una práctica muy útil, en relación con este aspecto, es la de revisar lo escrito desde la perspectiva del lector, a fin de determinar si es necesario, por ejemplo, el uso de ciertos "marcadores textuales", es decir, de frases organizativas con las cuales se da continuidad a lo que se escribe y se induce el sentido de las afirmaciones en el conjunto del texto producido.

\section{La segunda aduana: la construcción metodológica}

Quizá el punto más importante en un trabajo de investigación donde es posible determinar si existe articulación es la elección y configuración de un método de recopilación y análisis de datos, una vez que se ha presentado un objeto de estudio. Cervantes (1997) señala, sobre este punto, cómo las articulaciones que realiza un investigador o investigadora son resultado de operaciones cognoscitivas a partir de sus referencias conceptuales, de su 
avance de construcción del objeto y de su contacto con la realidad, lo cual le pone en una posición en la que es posible detectar las vinculaciones entre niveles y dimensiones planteadas en el fenómeno que estudia.

Una de las consecuencias de lo anterior es, entonces, la exigencia de trabajar, como indicaba Foucault (1973), en el esclarecimiento de las relaciones de la teoría con la experiencia, en una actitud descrita como "vigilancia epistemológica" por otros autores clásicos (Bachelard,1978; Bourdieu et al.,1975), donde se incluye la revisión rigurosa del método para detectar errores de construcción, siempre en atención a lo establecido en el marco conceptual de la investigación. Así mismo, en la medida de lo posible, esta actitud demanda del investigador o investigadora hacer explícitos los supuestos que le permiten formular sus interpretaciones en el momento de realizar el análisis de datos (Rodríguez, Lorenzo y Herrera, 2005).

La articulación objeto-método de investigación parte de la consideración lógica de la pertinencia entre lo que se pretende estudiar, generalmente expresado en una pregunta, los conceptos con los cuales se explica y los medios que se utilizan para buscar datos en la experiencia, con el fin de ofrecer respuestas a la pregunta construida. La metodología, por tanto, nunca se desconecta de la teoría, como señalan Bourdieu et al. (1975), y más recientemente, Stierer y Antoniou (2004), entre otros. Las técnicas e instrumentos no son neutrales, sino procedimientos que se integran en una perspectiva más amplia, de carácter teórico y conceptual, que da forma a la investigación completa.

De esta manera, en un trabajo articulado se espera encontrar una serie de afirmaciones en las cuales se conecta la teoría con el método, asumiendo que toda investigación tiene bases teóricas, conceptuales y metodológicas sobre las cuales se orienta (Ponce, Iglesias y Castillo, 2009). Las afirmaciones presentes en un trabajo de investigación son unidades muy importantes para analizar la existencia de esta articulación. En ellas puede verse, si quien produce el trabajo, tiene claras las implicaciones recíprocas entre objeto de estudio y método.

Por otra parte, la articulación, en la metodología de una investigación particular, refiere a expresar la relación entre componentes como el contexto o universo de estudio, los instrumentos, las fases del trabajo de campo y de presentación de resultados, entre otras cosas. Concretamente, en un trabajo articulado no solo se describen estos componentes, sino que se incluye una discusión de carácter epistemológico, que da cuenta de los conceptos teóricos fundamentales que sustentan la orientación desde la cual es concebido el objeto de estudio, de donde se desprende un acercamiento al campo con determinados procedimientos para la recolección y análisis de datos. De esta forma, es posible justificar cuestiones puntuales de orden metodológico, como por qué se emplean determinados instrumentos, cómo se utilizan y a quiénes se aplican, lo cual es frecuentemente poco sustentado en diversos trabajos de investigación.

En cuanto a esto último, cuando existen dudas sobre los aspectos particulares acerca de los cuales es necesario agregar explicaciones y realizar acomodos, durante la definición de la metodología y la producción de resultados en el análisis, la articulación se puede favorecer recurriendo precisamente a la pregunta de investigación principal. De esta manera, la atención a la pregunta de investigación proporciona la base para una articulación entre la metodología y el análisis de datos con los propósitos centrales de la investigación (Potter, 1998). 


\section{La tercera aduana. El análisis de los datos}

Los datos, en sentido general, son los resultados de la aplicación de ciertos instrumentos, junto con el significado que se les asigna a tales resultados, de acuerdo con el objeto de estudio. Por su parte, el análisis es entendido, en esta reflexión, siguiendo a Kerlinger y Lee (2001), como el conjunto de procedimientos sobre los datos, como la categorización y síntesis, los cuales se realizan con fines interpretativos. Al respecto, de acuerdo con estos autores, el investigador o investigadora debe trazar una ruta de análisis desde que inicia el trabajo con el objeto de estudio, lo cual puede incluir, en algunos casos, la formulación de hipótesis en torno a las preguntas de investigación.

De modo particular, cuando se establecen categorías para el tratamiento de los datos, si estas no se construyen de acuerdo con las demandas del objeto de estudio, entonces pueden existir respuestas inadecuadas a las preguntas. De ahí se tiene que también hay acciones que pueden resultar irrelevantes para dar respuesta a las preguntas mencionadas, como incluir categorías no congruentes o generar explicaciones sobre los datos que no son las requeridas, dado un objeto de investigación y una perspectiva teórica y empírica de estudio.

Durante la interpretación, se toman los datos y se hacen inferencias acerca de las relaciones de investigación planteadas en el objeto de estudio. Sin embargo, para que exista articulación entre este momento de la investigación y los demás, es necesario buscar el significado de los datos en el marco de las preguntas y los conceptos que dan forma al objeto de estudio, incluyendo los resultados de investigaciones relacionadas, las cuales por lo general se revisan al conformar el "estado de la cuestión”. De esta manera es posible dar mayor articulación a un producto de investigación, al realizar afirmaciones como las que establecen consistencia entre sus hallazgos con los de trabajos precedentes.

La interpretación de los datos justamente es uno de los aspectos de un trabajo de investigación en el cual pueden existir mayores discrepancias en la evaluación de los pares, debido a cuestiones relativas a la falta de articulación entre los datos presentados y las afirmaciones que forman parte de la interpretación. En este sentido, el cuidado de la correspondencia entre las aportaciones teóricas y las reflexiones del investigador o investigadora en torno al significado de los datos empíricos es un aspecto clave para lograr un trabajo articulado. Sobre este punto, autores como Coffey y Atkinson (2003) insisten en que el tratamiento analítico de los datos siempre debe estar penetrado por ideas teóricas.

Como consecuencia de lo anterior, una característica de un trabajo articulado es que no se produzcan "hallazgos" o interpretaciones fuera del marco teórico y metodológico que da sentido al análisis de los datos. De otra manera, aun cuando puedan ser valiosos, se están aportando elementos de respuesta a una pregunta distinta a la que se formuló y, con ello, se corre el riesgo de divagar en torno al objeto de estudio o de escindir partes del mismo que deberían estar vinculadas.

\section{A manera de conclusión}

La articulación, en cualquier trabajo escrito, pero en particular, en un producto de investigación, exige el señalamiento explícito de relaciones, de tal manera que se perciba una conjunción de elementos que comparten fines, tales como las piezas de una máquina o los instrumentos de una orquesta, más que como bloques de contenido superpuestos. Cuando ocurre esto último, el trabajo puede provocar la impresión de tratarse de una serie de "cajones" dispuestos en cierto orden, pero 
sin ensamble entre ellos. La articulación, así mismo, no solo hace referencia a la coherencia del documento, sino a la consistencia entre los momentos que van desde el planteamiento que se lleva a cabo durante la construcción del objeto, hasta la presentación de resultados y conclusiones.

Lograr la articulación, en el sentido que se ha señalado en este trabajo, lleva al investigador o investigadora a realizar una serie de acciones en torno a dos procesos: el primero tiene que ver con una comprensión epistemológica de lo que la investigación significa e incluye; es decir, de lo que supone el conocimiento en términos de su producción y validación; el segundo se refiere a dar cuenta de los procesos y los resultados en una investigación por medio de un producto escrito.

Del mismo modo, las debilidades de articulación que se pueden señalar en la crítica a un producto de investigación aluden a estos dos procesos. Como ejemplo, en el primer caso, puede cuestionarse la ausencia de reflexiones que muestren vínculos entre conceptos, procedimientos metodológicos y naturaleza de los datos presentes en una investigación. En el segundo, se puede señalar la falta de coherencia entre las subdivisiones creadas al interior de un texto para exponer los resultados y la estructura conceptual y metodológica precedente.

Sobre esto último conviene señalar que, por diversas circunstancias, a menudo, procesos de investigación como la búsqueda de bases teóricas, la construcción de perspectivas conceptuales y metodológicas o la recolección y procesamiento de los datos, ocurren de manera aislada o asincrónica. Esta anarquía; sin embargo, termina por ser solo temporal, puesto que la brújula que orienta el acomodo de tales procesos y de sus productos correspondientes es una sólida concepción teórica y una clara planeación metodológica. Se trata de construir una obra, de delinear una trama, una estructura propia donde los elementos encuentran su lugar desde la mirada de la persona que investiga. Como se ha señalado desde el inicio de esta reflexión, esa construcción atraviesa por el cumplimiento de dos criterios, uno de orden epistemológico y otro relacionado con características de la redacción académica. De allí que sea muy importante, para conseguir articulación en un trabajo de investigación:

1. Concebir la investigación como un todo donde cada parte permite dar sentido a las demás, y donde teoría y metodología se implican mutuamente.

2. Revisar la coherencia entre las unidades de comunicación de las ideas (conceptosafirmaciones-párrafos-apartados-capítulos-texto completo).

Lo anterior exige también profundizar en el conocimiento de las nociones de la disciplina en que se sitúa el investigador o investigadora, donde privan ciertos conceptos teóricos que son base de las tradiciones de estudio del fenómeno que le interesa estudiar. Para ello, quien investiga requiere tener conocimiento de las concepciones más amplias y generales acerca de la realidad y del hombre mismo, de los métodos que deben emplearse para abordarla y de las maneras legítimas de plantear cuestiones. 


\section{Referencias}

Bachelard, G. (1978). La formación del espíritu científico. Contribución a un psicoanálisis del conocimiento objetivo. México: Siglo XXI.

Bourdieu, P., Chamboredon, J.-C. y Passeron, J.-C. (1975). El oficio del sociólogo. Presupuestos epistemológicos. México: Siglo XXI.

Cassany, D. y Morales, O. A. (enero-diciembre, 2008). Leer y escribir en la universidad: Hacia la lectura y la escritura crítica de géneros científicos. Memoralia, 5, 69-82, Recuperado de http:// www.falemosportugues.com/pdf/leer_universidad.pdf

Cervantes, C. (1997, mayo-agosto). Problematizar la "articulación" como recurso metodológico utilizado en el campo académico de la comunicación en México. Comunicación y Sociedad, 30, 79-99. Recuperado de http://www.publicaciones.cucsh.udg.mx/pperiod/comsoc/ pdf/30_1997/79-99.pdf

Coffey, A. y Atkinson, P. (2003). Encontrar el sentido a los datos cualitativos. Estrategias complementarias de investigación. Colombia: Universidad de Antioquia.

Foucault, M. (1973). El orden del discurso. Buenos Aires: Tusquets.

Kalman, J. (septiembre, 2000). La importancia del contexto. Conferencia magistral presentada en el Día Internacional de la Alfabetización llevado a cabo en Morelia, Michoacán, México.

Kerlinger, F. y Lee, H. (2001). Investigación del comportamiento: Métodos de investigación en ciencias sociales. México: McGraw-Hill.

Narvaja de Arnoux, E., Di Stéfano, M. y Pereira, C. (2002). La lectura y la escritura en la universidad. Buenos Aires: Eudeba.

Ponce, H., Iglesias, R. y Castillo, R. (2009). Estrategia metodológica para desarrollar proyectos de investigación en ciencias sociales. Recuperado de http://www.eumed.net/rev/cccss/06/tab.htm

Potter, J. (1998). Crítica de hechos. En J. Potter (Aut.). La representación de la realidad. Discurso, retórica y construcción social (pp. 255-292). Barcelona: Paidós.

Rodríguez, C., Lorenzo, O. y Herrera, L. (2005, julio-diciembre). Teoría y práctica del análisis de datos cualitativos. Proceso general y criterios de calidad. Revista Internacional de Ciencias Sociales y Humanidades SOCIOTAM, 15(2), 133-154. 
Stierer, B. y Antoniou, M. (2004, julio). Are there Distinctive Methodologies for Pedagogic Research in Higher Education? [¿Existen metodologías distintivas para la investigación pedagógica en educación superior?]. Teaching in Higher Education, 9(3), 275-285.

Zanotto, M. (2007). Estrategias de lectura en lectores expertos para la producción de textos académicos (Tesis doctoral). Departamento de psicología básica, evolutiva y de la educación, Universidad Autónoma de Barcelona, Recuperada de http://www.tesisenred.net/bitstream/ handle/10803/4759/mzg1de1.pdf?sequence $=1$ 